\title{
Letter to the Editor concerning "Low virulence bacterial infections in cervical intervertebral discs: a prospective case series" by Chen Y, Wang X, Zhang X, et al. (Eur Spine J; 2018: doi:10.1007/ s00586-018-5582-4)
}

\author{
Manu N. Capoor ${ }^{1,4}\left[\right.$ (D) Andrew McDowell ${ }^{2} \cdot$ Assaf Raz $^{1} \cdot$ Peter Lambert $^{3} \cdot$ Ondrej Slaby $^{4}$
}

Received: 22 May 2018 / Accepted: 17 June 2018

(c) The Author(s) 2018

\section{Dear Editor,}

We have read with great interest the manuscript by Chen et al., which provides an interesting and potentially important perspective into low virulence infections in cervical intervertebral discs [1]. Given our experience in relation to the study of infection amongst patients undergoing lumbar microdiscectomy surgery [2], we would like to highlight what we consider to be some methodological and data analysis limitations within the study that could impact on the final results and conclusion. These are:

1. The lack of a homogenization step in the processing of the retrieved disc material, which was only cut into small pieces before enrichment culture. Our previous study based on fluorescence in situ hybridization confocal scanning laser microscopy has shown that Propionibacterium acnes is present deep within intervertebral disc tissue as a biofilm (Fig. 1) and, as a consequence, it is imperative that the biofilm is disrupted prior to culture to maximize detection and reduce the possibility of a false-negative result [2]. This can be achieved

Manu N. Capoor

mcapoor@ rockefeller.edu

$\bowtie$ Ondrej Slaby

ondrej.slaby@ceitec.muni.cz

1 Laboratory of Bacterial Pathogenesis and Immunology, Rockefeller University, New York, NY, USA

2 Northern Ireland Centre for Stratified Medicine, School of Biomedical Sciences, Ulster University, Londonderry, UK

3 School of Life and Health Sciences, Aston University, Aston Triangle, Birmingham, UK

4 Central European Institute of Technology (CEITEC), Masaryk University, Brno, Czech Republic by homogenization with a mortar and pestle, with the option of a short sonication step. In contrast, there was no evidence of CoNS present in disc tissue as a biofilm. The process of disrupting $P$. acnes biofilm is a recognized approach to maximize the detection of $P$. acnes and CoNS organisms associated with other infections, notably those associated with prosthetic joints [3].

2. The study population is underpowered based on a sample size calculation: Two meta-analyses of previous studies addressing infection of intervertebral discs reported a pooled prevalence of bacteria at 34 and $36.2 \%$, respectively, with $P$. acnes as the predominant species $[4,5]$. An appropriate sample size estimation should therefore be calculated based upon these prevalence rates [sample size $\left.=\left(1.96^{2} \times \mathrm{PR}(1-\mathrm{PR})\right) / 0.05^{2}\right) ;$ note $\mathrm{PR}=$ prevalence rate] [6]. To ensure that the $95 \%$ confidence interval estimate of the proportion positive cases is within 5\% of the true proportion, a sample size of approximately 350 cases is necessary. Indeed, in our previous work we looked at 368 patients to draw statistically relevant conclusions [2].

3. Directly comparing the prevalence of a single species like $P$. acnes to a genus level grouping such as coagulase-negative staphylococci (CoNS) is inappropriate in the context of assessing potential clinical relevance. Such an analysis of recovery rates could undervalue the significance of $P$. acnes as a standalone species, and skew statistical significance (Table 1). As an exemplar of this, in our previous study of 368 subjects we found $P$. acnes present in 119 cultured intervertebral disc specimens versus 61 CoNS (scenario 1); in the latter case, $S$. epidermidis, which was the most common CoNS species, was only present in 15 cases (scenario 2) [2]. In scenario 1, the total prevalence or incidence of $P$. acnes was therefore $66 \%$, whilst in scenario 2 it was $89 \%$. 

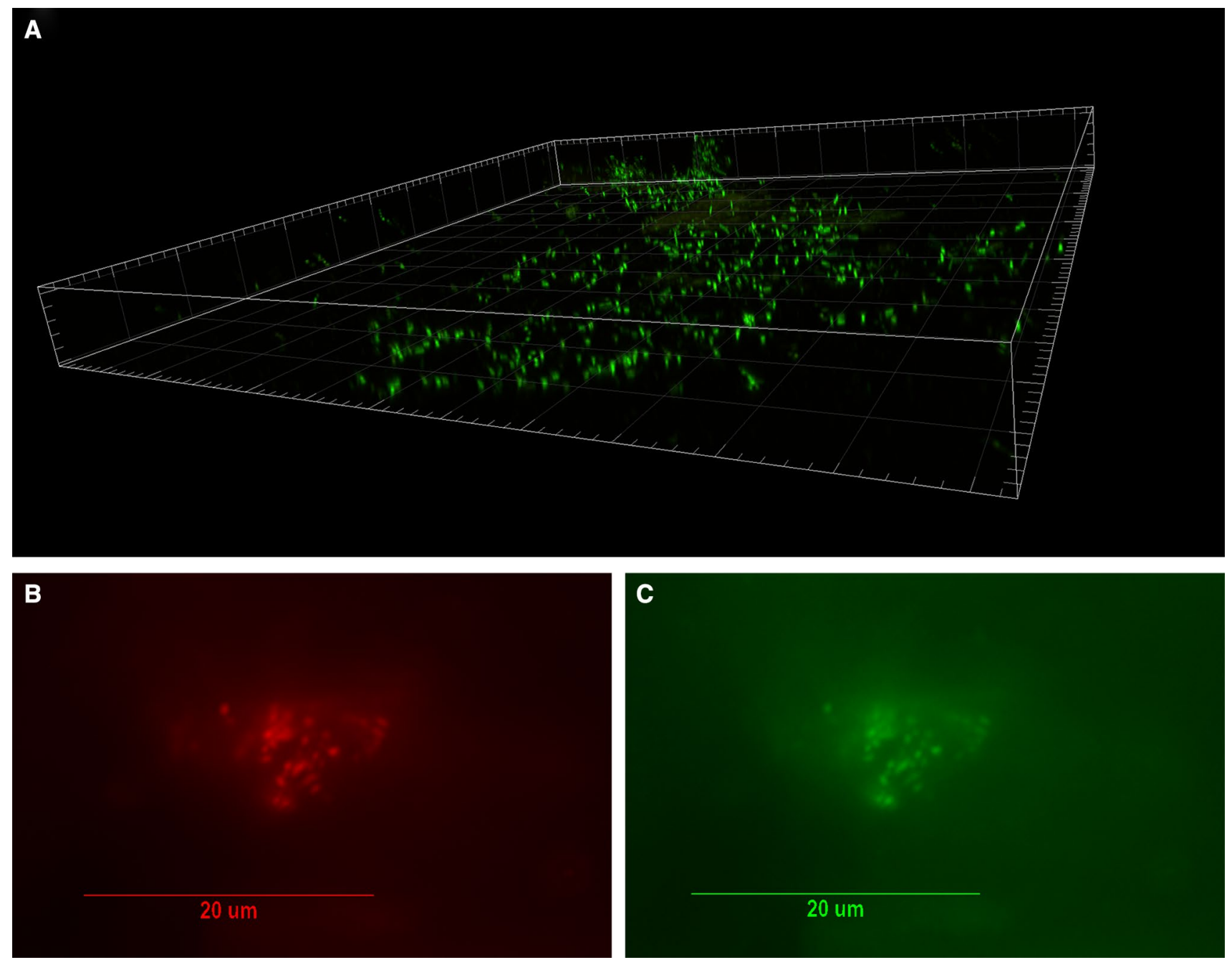

Fig. 1 Visualization of bacterial biofilm in the disc tissue by CSLM and confirmation of $P$. acnes by FISH (Reprinted from Capoor et al. 2017). a Three-dimensional reconstructed CSLM image of biofilm bacteria stained with a DNA stain (SYTO9, green) in a disc tissue sample. $\mathbf{b}$, $\mathbf{c}$ The presence of $P$. acnes biofilms in this sample verified

using FISH. Epifluorescence micrographs of a biofilm cluster showing red fluorescence from the CY5-labelled EUB338 general eubacterial probe (b) and green fluorescence from the CY3-labelled P. acnesspecific probe (c). Co-localization of the red and green fluorescence indicates that all of the bacteria in this biofilm were $P$. acnes

This $23 \%$ difference was statistically very significant $(p<0.001)$. Similarly, in the study of Chen et al. the incidence of $P$. acnes changes from $20 \%$ when CoNS are grouped together and considered, to $40 \%$ when only S. epidermidis is analysed as a comparator species. This

large difference of $20 \%$ did not reach statistical significance, however, due to the very small isolate numbers involved $(p=0.604)$.

4. The use of enrichment culture, whilst a common approach in these types of study, is particularly liable
Table 1 Incidence of $P$. acnes recovery from intervertebral discs based on comparison with CoNS or S. epidermidis

\begin{tabular}{lllll}
\hline Study & P. acnes versus CoNS & $\begin{array}{l}\text { P. acnes versus } \\
\text { S. epidermidis }\end{array}$ & $\begin{array}{l}\text { Difference in } P . \\
\text { acnes incidence }\end{array}$ & $p$ value (95\% CI) \\
\hline Capoor et al. [2] & 119 versus 61 & 119 versus 15 & $23 \%(66$ vs $89 \%)$ & $<0.001(13.8-31.4)$ \\
Chen et al. [1] & 2 versus 7 & 2 versus 3 & $20 \%(20$ vs $40 \%)$ & $0.604(-23.1$ to 59.7) \\
\hline
\end{tabular}

$S$. epidermidis were the most frequent non- $P$. acnes bacteria in both studies. Chen et al. had 8 positive cases of which 2 were $P$. acnes, 1 was $S$. capitis, 2 were $S$. haemolyticus, 2 were $S$. epidermidis, and 1 case was $S$. capitis and $S$. epidermidis. Capoor et al.'s bacterial breakdown by case is shown in Fig. 2. $p$ values for comparison of proportions were calculated using the $\mathrm{N}-1$ Chi-squared test 
Fig. 2 Microorganisms isolated in 162 cases from 368 patients undergoing microdiscectomies. Bacterial growth was observed with 162 of 368 specimens (44\%); no colonies were observed with the other 206. Propionibacterium acnes was present in 119 (32.3\%), and $S$. epidermidis, the most frequent other bacterial species, occurred in 15 cases $(4.1 \%)$

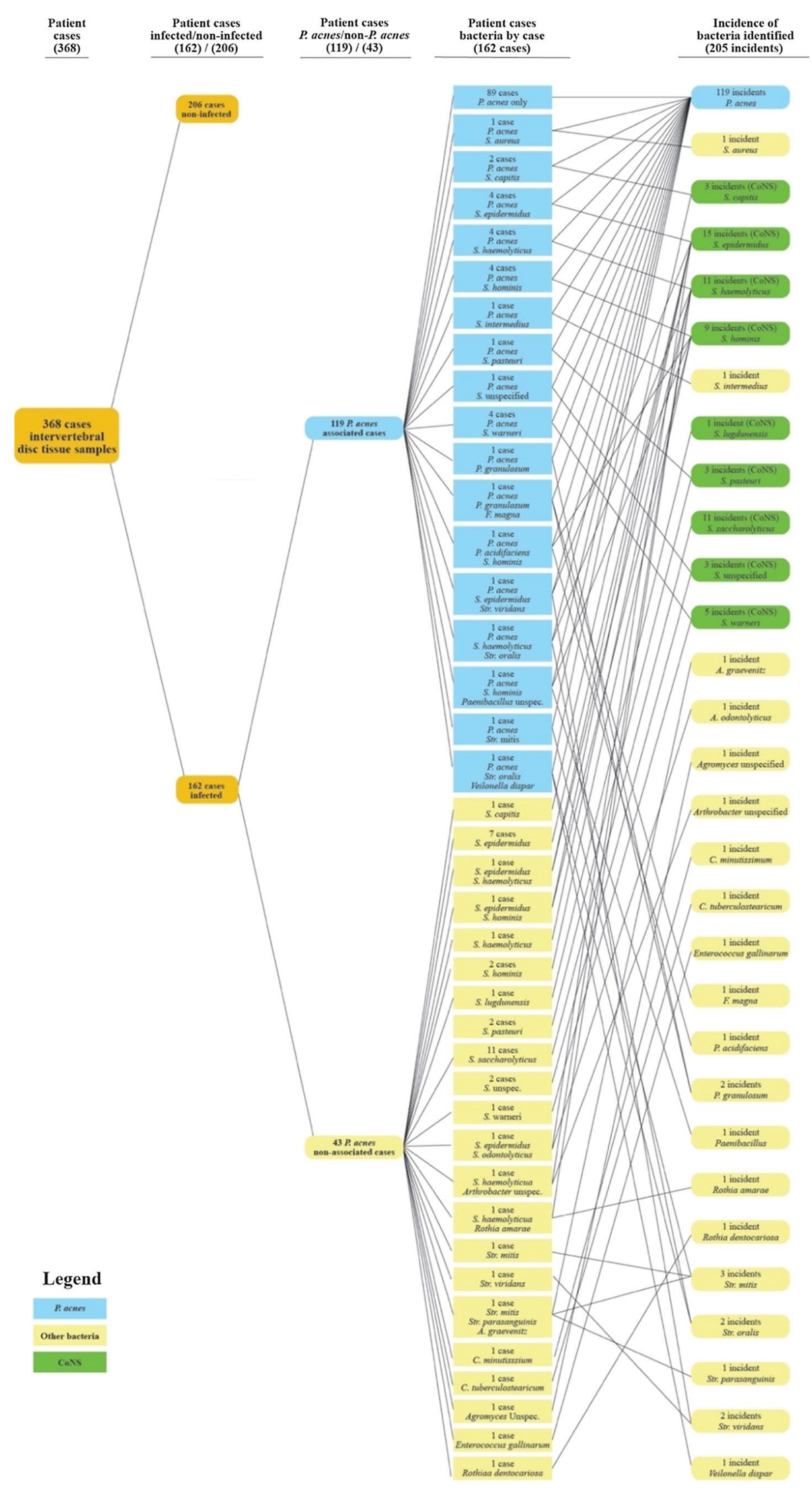


to contamination unless scrupulous aseptic technique is adhered to. Furthermore, in a mixed sample of CoNs and $P$. acnes, even when grown anaerobically, the doubling time of Staphylococci (approx. $100 \mathrm{~min}$ ) is still much greater than that of $P$. acnes (approx. $300 \mathrm{~min}$ ). This disparity in growth rate may cause most Staphylococcus to overwhelm $P$. acnes growth thereby creating a biased result $[7,8]$. Culture on aerobic and anaerobic blood agar plates is a much better approach, as long as the tissue is homogenized.

5. Although $P$. acnes is aerotolerant, a culture approach or methodology similar to that used for strict anaerobes is best practice to maximize recovery from clinical samples, especially if counts are low. Clinical samples should be transferred immediately from the surgical theatre to the laboratory under an anaerobic atmosphere, and all growth media and diluents should be pre-reduced and pre-equilibrated under anaerobic conditions prior to processing. All liquid media and diluents should also contain a suitable reducing agent like L-cysteine hydrochloride $(0.05 \% \mathrm{w} / \mathrm{v})$, and any processing should be carried out in an anaerobic cabinet. It is unclear the extent to which such protocols were followed by Chen et al., and indeed others researchers working on the role of infection in degenerative disc disease, but this is best practice and very important when attempting to determine the rate of recovery of an organism like $P$. acnes.

The study of Chen et al. was focused on the analysis of cervical discs, and in this context it is unclear if direct comparison with other studies which have focused on lumber disc degeneration is completely appropriate in terms of the rate of recovery of $P$. acnes and other bacterial organisms $[2,9]$. Nonetheless, if an attempt is to be made to investigate the prevalence of low virulence infection, such as $P$. acnes, with cervical discs, then we feel the methodological and data analysis issues described here may have compromised any clear conclusions, especially as $P$. acnes does appear to be significantly represented within infected discs [2].

\section{Compliance with ethical standards}

Conflict of interest MNC, AR, and OS have shares or options in DiscitisDx. AM and PL have no conflicts.

Open Access This article is distributed under the terms of the Creative Commons Attribution 4.0 International License (http://creativecommons.org/licenses/by/4.0/), which permits unrestricted use, distribution, and reproduction in any medium, provided you give appropriate credit to the original author(s) and the source, provide a link to the Creative Commons license, and indicate if changes were made.

\section{References}

1. Chen Y, Wang X, Zhang X, Ren H, Huang B, Chen J, Liu J, Shan Z, Zhu Z, Zhao F (2018) Low virulence bacterial infections in cervical intervertebral discs: a prospective case series. Eur Spine J. https://doi.org/10.1007/s00586-018-5582-4

2. Capoor MN et al (2017) Propionibacterium acnes biofilm is present in intervertebral discs of patients undergoing microdiscectomy. PLoS ONE 12(4):e0174518

3. Tunney MM et al (1998) Improved detection of infection in hip replacements. A currently underestimated problem. J Bone Joint Surg Br 80(4):568-572

4. Urquhart DM et al (2015) Could low grade bacterial infection contribute to low back pain? A systematic review. BMC Med 3:13

5. Ganko R et al (2015) Can bacterial infection by low virulent organisms be a plausible cause for symptomatic disc degeneration? A systematic review. Spine 40(10):E587-E592

6. Naing $L$ et al (2006) Practical issues in calculating the sample size for prevalence studies. Arch Orofac Sci 1:9-14

7. Hall GS et al (1994) Growth curve for Propionibacterium acnes. Curr Eye Res 13(6):465-466

8. Belay N, Rasooly A (2002) Staphylococcus aureus growth and enterotoxin A production in an anaerobic environment. J Food Prot 65:199-204

9. Stirling A, Worthington T, Rafiq M, Lambert PA, Elliott TS (2001) Association between sciatica and Propionibacterium acnes. Lancet 357(9273):2024-2025 